INFORMATIONSTECHNOLOGIE

\title{
Report bringt Licht ins Dunkel
}

\section{Helmut Kreidenweis und Bernd Halfar}

\begin{abstract}
Die Sozialwirtschaft in Deutschland ist Pionier bei der Nutzung moderner Technik: Rund 80 Prozent der Beschäftigten in sozialen Diensten und Einrichtungen arbeiten in irgendeiner Form mit einem Arbeitsplatz-Computer. Dies ist eines der überraschenden Ergebnisse des ersten Reports zum Einsatz von Informationstechnologie in sozialen Organisationen.
\end{abstract}

In vielen Branchen ist es ein vertrautes Ritual: Kennzahlen zum Einsatz von Informationstechnologie (IT) werden regelmäßig erhoben, publiziert und in der Fachwelt eifrig diskutiert. Die Sozialwirtschaft wusste bislang fast nichts darüber, in welchem Umfang, auf welche Art, mit welchen Budgets oder Sicherheitsstandards IT eingesetzt wird. Auch über den Anbietermarkt von Branchensoftware wurden seit Ende der 1990er Jahre keine systematischen Informationen mehr erhobenen, Kennzahlen aus diesem Sektor gab es bislang keine. Dabei braucht die deutsche Sozialwirtschaft mit rund 80 Milliarden Euro Jahresumsatz und weit über einer Million Beschäftigten den Volumenvergleich mit vielen klassischen Industrie- oder Dienstleistungsbranchen nicht zu scheuen.

Der bundesweit erste IT-Report für die Sozialwirtschaft, den die Arbeitsstelle für Sozialinformatik an der Katholischen Universität Eichstätt-Ingolstadt nun publiziert hat, versucht Licht in dieses Dunkel zu bringen. Dieser Beitrag stellt einige ausgewählte Ergebnisse dieser Untersuchung vor. Der komplette IT-Report kann bei der Katholischen Universität bezogen werden (vgl. Kasten Seite 27). Die Studie basiert auf zwei schriftli- chen Befragungen, die im August und September 2007 durchgeführt wurden. Zielgruppen waren zum einen soziale Organisationen als Anwender von Informationstechnologien und zum anderen Anbieter von IT-Lösungen für die Sozialwirtschaft. Beide Befragungen waren auf das Gebiet der Bundesrepublik Deutschland begrenzt.

\section{Ergebnisse}

Zielgruppe der Untersuchung im Bereich der sozialen Organisationen waren Einzel- und Komplex-Einrichtungen sowie Trägerverbände. Neben der freien Wohlfahrtspflege wurden auch kommunale und privat-gewerbliche Anbieter sozialer Dienstleistungen angesprochen. Es wurden 950 Fragebögen verschickt, die Rücklaufquote betrug 8,6 Prozent. Die Stichprobe bestand überwiegend aus Organisationen mit mehr als 200 Mitarbeiterinnen und Mitarbeitern, da vielfach nur sie über Ansprechpartner verfügen, die in der Lage sind, die komplexen Fragestellungen zu beantworten. Damit spiegeln die Ergebnisse nicht die gesamte heterogene sozialwirtschaftliche Landschaft wieder, sondern lenken den Blick vor allem auf die Großen des Marktes. Bei ihnen handelt es sich vielfach um Anbieter mehrerer Hilfearten oder um Komplexträger.

Mit einer durchschnittlichen Mitarbeiterzahl von 883 und einer Platzbzw. Bettenzahl von 1.249 in durchschnittlich 25 Standorten bringen es die befragten Unternehmen im Schnitt auf einen Umsatz in Höhe von 41 Millionen Euro. Die Streubreite ist hier allerdings groß. Durchschnittlich setzen diese Organisatio-

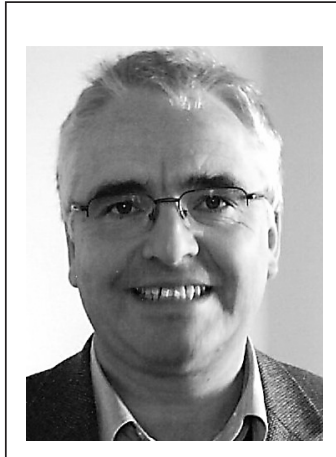

Prof. Dr.

Bernd

Halfar

lehrt Ma-

nagement

in sozialen

Einrich-

tungen/

Organisa-

tionsent-

wicklung an der Katholischen Universität Eichstätt-Ingolstadt und ist Partner der Unternehmensberatung xit.forschen. planen.beraten, Nürnberg E-Mail bernd.halfar@kueichstaett.de

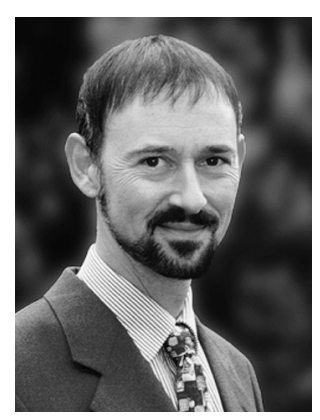

Prof. Helmut Kreidenweis lehrt Sozialinformatik an der $\mathrm{Ka}$ tholischen Universität Eichstätt-Ingolstadt und ist Inhaber von KI Consult, Augsburg IT-Beratung für soziale Organisationen.

E-Mail helmut.kreidenweis@ ku-eichstaett.de

nen etwa ein Prozent ihres Umsatzes für Informationstechnologie ein. Vorherrschender Rechnertyp ist mit 73 Prozent noch immer der klassische Schreibtisch-PC, Notebooks und die modernen Thin Clients für serverbasierte Netzwerke sind noch deutlich in der Minderheit.

Erstaunlich ist, dass in den befragten Organisationen im Durchschnitt bereits 80 Prozent der Mitarbeitenden in irgendeiner Form Nutzer von Arbeitsplatz-Computern sind. Damit liegt die Sozialwirtschaft weit über dem Durchschnittswert aller Branchen in Deutschland (61\%). Selbst Finnland, mit 70 Prozent Spitzenreiter in der Europäischen Union, wird damit von der deutschen Sozialwirtschaft übertroffen. Dennoch hat hier längst nicht jeder Mitarbeiter einen eigenen PC: Im Mittel kommen auf einen IT-Arbeitsplatz 4,7 Mitarbeiter, was auch die Spezifika der Arbeitsge- 
staltung in den pflegerischen und pädagogischen Dienstleistungsbereichen widerspiegelt.

\section{Infrastruktur}

Netzwerke haben längst den klassischen Stand-Alone-PC abgelöst, durchschnittlich sind heute 92 Prozent der Rechner vernetzt. Unter den Betriebssystemen dominiert erwartungsgemäß Windows, jedoch scheint Linux im Kommen zu sein: Immerhin fast ein Viertel der Organisationen setzen das Open-Source-Betriebssystem bereits im Serverbereich ein. An den Arbeitsplätzen ist noch immer fast die Hälfte der mit Windows ausgestatteten Rechner mit Vorgängerversionen des 2001 erschienenen Windows XP ausgestattet.

Entsprechend dürfte auch das Alter der zugehörigen Hardware datierbar sein, was auf durchschnittliche Nutzungszeiten von deutlich über fünf Jahren hindeutet (vgl. Grafik »ServerEinsatz nach Funktionen $\ll)$.

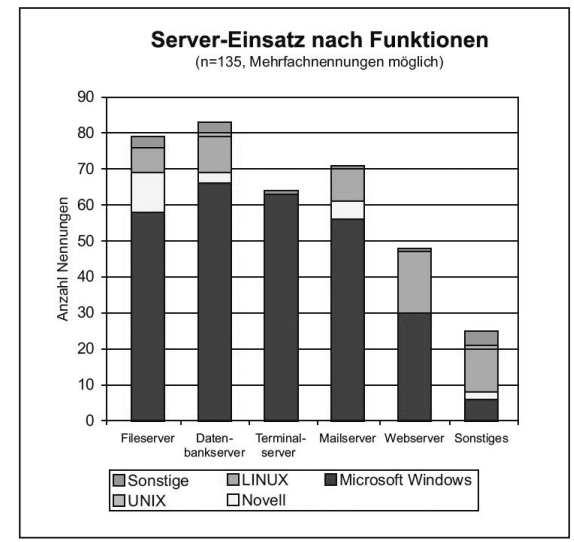

Im Bereich fachspezifischer Programme für Leistungsabrechnung, Planung und Dokumentation von Hilfen oder Dienstplanung setzen soziale Organisationen heute vorwiegend auf Fremdsoftware einschlägiger Branchen-Anbieter. Unter den Anbietern dominieren in den meisten Feldern die Firmen All vor One, Micos, Connext, develop group Sigma sowie TDS. Open Source Software wird bislang nur zögerlich implementiert, nur ein Fünftel nutzt überhaupt solche Programme.

\section{Organisation und Personal}

IT-Organisation, das bedeutete in vielen sozialen Organisationen lange Zeit die Existenz eines »EDV-Beauftragten«, der mit Blaumann und Schau- benzieher bewaffnet durch die Gänge trottete, immer auf der Jagd nach lockeren Steckern und leeren Druckerpatronen. Heute hat von den untersuchten Organisationen immerhin noch ein Viertel die Gesamtverantwortung für die Informationstechnologie noch nicht institutionalisiert und jede 20. hat diese komplett nach außen gegeben. Die andern haben jeweils etwa zur Hälfte einen IT-Leiter mit eigener Stelle oder diese Aufgabe mit einer anderen Funktion kombiniert.

Das IT-Personal besteht nur zum Teil aus spezialisierten Fulltime-Kräften, Aufgaben aus diesem Bereich werden häufig auch von Praktikanten, Azubis, Zivis oder festen Mitarbeitern mit eher geringen IT-Stellenanteil erledigt. Rechnet man all diese Varianten auf Vollzeitstellen hoch, so steht eine IT-Stelle durchschnittlich 132 IT-Anwendern gegenüber. Die Unterschiede zwischen den Organisationen sind hier jedoch groß.

\section{Sicherheit und Datenschutz}

Die Sozialbranche gilt als besonders sensibles Feld bei der Erhebung und Speicherung personenbezogener Daten. Wahrscheinlich gehören die hier untersuchten Unternehmen hinsichtlich ihres Professionalisierungsgrades in der Informationstechnologie eher zur »Crème« der Branche. Vier von fünf dieser Unternehmen haben ein IT-Sicherheitskonzept, 87 Prozent einen schriftlich bestellten Datenschutzbeauftragten. Risse im Bild einer wohl organisierten IT- Sicherheit zeigen sich $\mathrm{u}$. a. bei der Frage nach der Anwendung standardisierter Sicherheitsnormen. Nur 35 Prozent richten sich danach und 38 Prozent ist die IT-Sicherheit maximal fünf Prozent ihres IT-Budgets wert (vgl. Grafik »Anteil der Aufwendungen für IT-Sicherheit am IT-Budget «).

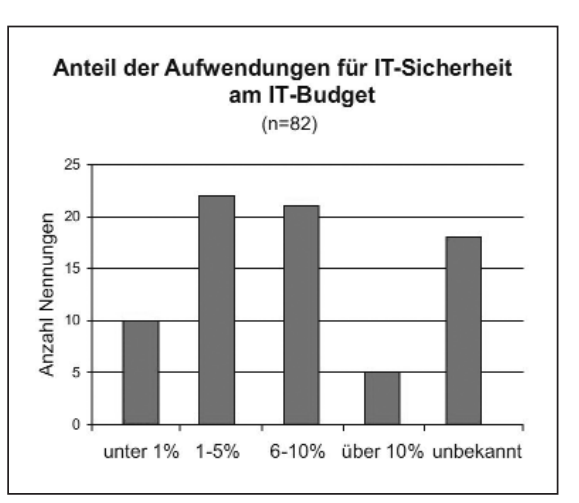

\section{Budget und Investitionen}

Über ein vorab geplantes und vereinbartes IT-Budget verfügen drei Viertel der Unternehmen. Schwerpunkte für Investitionen innerhalb der kommenden zwölf Monate bilden die Bereiche Branchen-Software und Hardware. Innerhalb des Bereichs Branchensoftware stehen Investitionen in Programme für Planung und Dokumentation von Hilfen an erster Stelle. Insgesamt ist die Investitionsbereitschaft in Branchensoftware durchaus hoch, die Anbieter können sich darauf einstellen, dass die Karten vielfach neu gemischt werden.

\section{Sicht der IT-Verantwortlichen}

Die IT-Verantwortlichen sind insgesamt mit ihrer Ausstattung in erstaunlich hohem Maße zufrieden, die technische Ausstattung liegt in auf dieser Skala jedoch klar vor der personellen. Entwicklungsbedarfe sehen sie vor allem in der Standardisierung und Zentralisierung ihrer IT-Landschaften. Ebenso Priorität genießt die IT-Qualifikation der Mitarbeiterschaft. Als Trend bei der Branchensoftware erwarten die ITVerantwortlichen unter anderem eine höhere Integration oder Vernetzung der Programme und Funktionalitäten.

\section{Der Branchensoftware-Markt}

Kriterium für die Aufnahme von Anbietern von IT-Lösungen in den IT-Report war, dass sie branchenspezifische Software für soziale Einrichtungen und Verbände entwickeln oder vertreiben. Von den knapp 300 verschickten Fragebögen kamen 75 ausgefüllt zurück. Der SoftwareAnbietermarkt für die Sozialwirtschaft ist stark von Unternehmensstrukturen geprägt, die am unteren Skalenrand dessen stehen, was gemeinhin als »mittelständisch « bezeichnet wird. Im Unterschied zur starken Marktkonzentration vielen anderen Softwaremärkten ist dieser Bereich noch immer stark zersplittert. Mehrjahresvergleiche in den künftigen Ausgaben des IT-Reports werden zeigen, ob hier ein spürbarer Konzentrationsprozess einsetzt.

Die 75 antwortenden Unternehmen beschäftigen zusammengenom- 
men circa 1.400 Mitarbeiterinnen und Mitarbeiter im Geschäftsfeld Sozialwirtschaft. Schätzt man die Anzahl der derzeit deutschlandweit marktrelevanten Anbieter von Branchenlösungen auf etwa 120 Firmen, so ergibt sich hochgerechnet eine Anzahl von über 2.200 Arbeitsplätzen, die durch Entwicklung, Support und Vertrieb von Software für das Sozialwesen in Deutschland gesichert werden. Ein Beispiel für die Beschäftigungswirkung für »Zulieferindustrien« des sozialen Sektors, die bislang kaum öffentlich wahrgenommen wird (vgl. Grafik »Gesamtzahl der Mitarbeiter «). Hochgerechnet beträgt der Jahresumsatz mit sozialwirtschaftlichen IT-Lösungen insgesamt gut 140 Millionen Euro. Dies entspricht etwa dem Umsatz der SAP AG mit Softwarelizenzen in den Vereinigten Staaten (2005).

Im Mittel bedienen die befragten Unternehmen 432 Kunden im sozialwirtschaftlichen Bereich. Die Spannbreite reicht jedoch von einem bis $\mathrm{zu}$ mehreren tausend. Die Hälfte der Anbieter hat ihre Software bei weniger als fünf Arbeitsplätzen pro Kunde im Einsatz.

In der Sozialwirtschaft setzen die befragten Unternehmen im Schnitt gut 1,7 Millionen Euro um, auch hier zeigt sich eine enorme Spannbreite. Der durchschnittliche Umsatz pro Mitarbeiter beträgt hier gut 80.000 Euro. Vergleicht man diese Kennzahlen mit großen Software-Unternehmen in Deutschland, so werden Unterschiede sichtbar: So setzt die SAP AG etwa 132.000 Euro pro Mitarbeiter um und die Sage (KHK) Software GmbH 120.000 Euro.

Im Ranking nach Umsatzzahlen im sozialwirtschaftlichen Geschäftsfeld liegen die Firmen All for One, Connext und Micos an der Spitze, Rankings nach Mitarbeiter- oder Kundenzahlen zeigen teils andere Ergebnisse, die sich aus unterschiedlichen Geschäftsmodellen oder transnationalen Firmenaktivitäten erklären.

Mit dem Geschäftsverlauf der zurückliegenden zwölf Monate sind fast zwei Drittel der befragten IT-Anbieter zufrieden. Noch etwas positiver als der Rückblick fallen die Umsatzerwartungen für die kommenden zwölf Monate aus: Mehr als drei Viertel erwarten einen Anstieg. Bei ihren Investitionen räumen die Anbieter dem

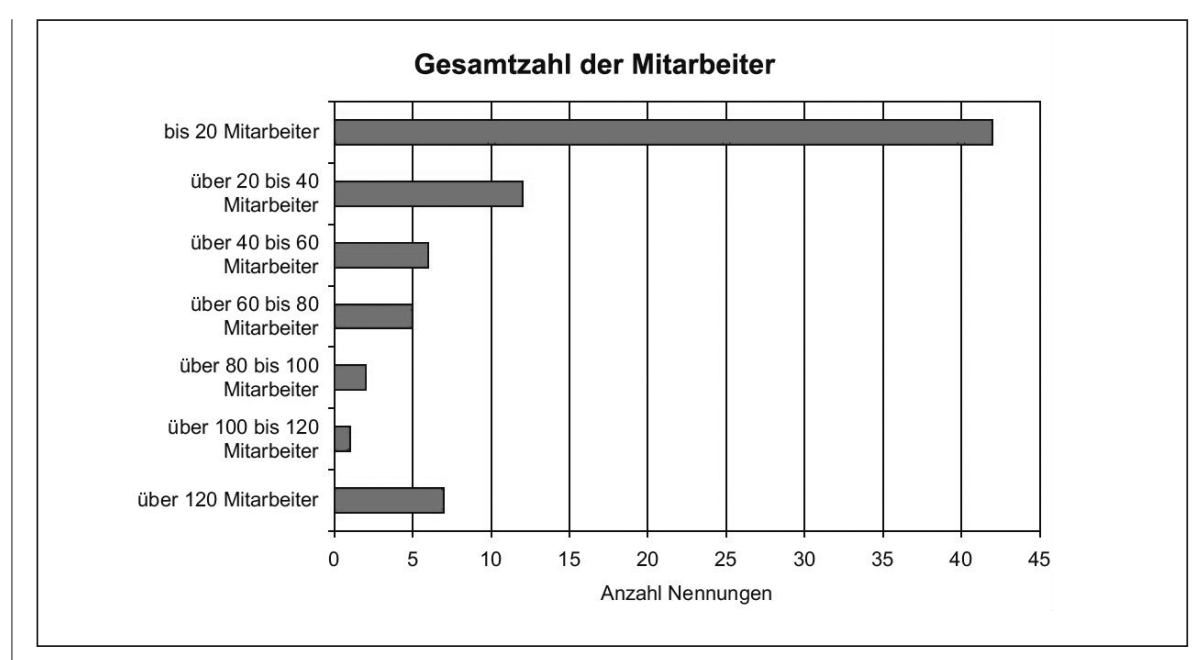

funktionellen Ausbau vorhandener Programme den Vorrang ein. Die stärkste Nachfrage erwarten sie im kommenden Jahr in den Bereichen Informations- und Wissensmanagement sowie Dokumentenmanagement und Archivierung. Als Zukunftstrends werden $u$. a. eine vermehrte Vernetzung über Einrichtungsgrenzen hinweg sowie den verstärkten Einsatz von Web-Technologien gesehen.

Ob sich der optimistische Ausblick der IT-Anbieter bestätigen wird? Die Anbieterlandschaft im Sozialmarkt ist merk-würdig. Branchenriesen wie SAP oder Microsoft tun sich offensichtlich immer noch sehr schwer, in diesen Markt nennenswert Einlass zu finden. In der derzeitigen Anbieter-
Landschaft stehen zahlreichen Kleinunternehmen mit hoch spezialisierter Fachsoftware einer übersichtlichen Zahl von eher mittelständisch geprägten Unternehmen gegenüber, die meist arbeitsfeldübergreifende Lösungen anbieten. Das von Nachfragerseite geäußerte Interesse an WorkflowSystemen, einer stärkeren

Einbindung von Internettechnologien oder der Integration der IT-Systeme stammen weitgehend aus größeren Organisationen. Ob und wie sich die Arbeitsteilung zwischen $» \mathrm{Ni}$ schenlösungen « und »Komplettangeboten « organisieren wird, muss die Zukunft zeigen. Die im Jahresrhythmus erscheinenden Ausgaben des ITReports werden Antworten auf diese Fragen geben.
Bernd Halfar und Helmut Kreidenweis

\section{IT-Report für die Sozialwirtschaft 2007/2008}

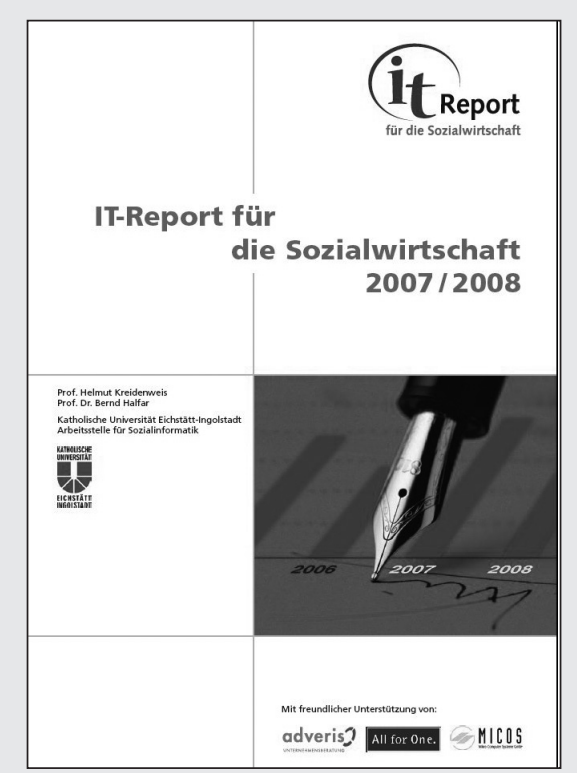

Der IT-Report enthält über 30 Charts und 10 Tabellen mit detaillierten Angaben zu allen untersuchten Punkten. Weiterhin ist Verzeichnis mit über 70 Anbietern von Fachsoftware für soziale Organisationen mit detaillierten Firmen- und Produktinformationen enthalten. Inhaltsverzeichnis und Beispielseiten sind unter www.sozialinformatik.de einsehbar.

Bezug: Katholische Universität Eichstätt Ingolstadt, Arbeitsstelle für Sozialinformatik Telefon 08421 93-1472

E-Mail

christine.vetter@ku-eichstaett.de

Preis 50,- Euro zuzüglich Versandkosten (Versand gegen Rechnung) 